\title{
A LARGE AREA THOMSON-SCATTERING STELLAR X-RAY POLARIMETER*
}

\author{
R. NOVICK and R. S. WOLFF \\ Columbia Astrophysics Laboratory, Columbia University, New York, N.Y., U.S.A.
}

\begin{abstract}
An instrument for measuring the polarization of stellar X-ray emission has been designed, constructed, and tested. The polarization dependence of incoherent Thomson scattering in lithium metal is utilized. Means for suppression of cosmic ray background effects have been provided. The apparatus has been flown in rockets to obtain data in the polarization of X-ray sources in Sco X-1 and Tau X-1.
\end{abstract}

The study of radio and visible light polarization has played a significant and often decisive role in the development of our understanding of astrophysical processes. The observation of the spatial, temporal and spectral distribution of polarization has often allowed one to uniquely specify the emission process. In the case of the Crab Nebula, the observation of large-scale quasi-homogeneous radio and optical polarization provided clear-cut evidence for the synchrotron mechanism; in M 82 recent unpublished polarization data suggest that electron scattering of the radiation is occurring in the outer regions of the object; in the Crab pulsar NPO 532 the radio and optical pulses show a tantalizingly simple temporal behavior whose elucidation will almost certainly lead to a full understanding of the pulsar emission process. Finally, the observation of highly polarized knots in M 87 suggests that the nucleus of this galaxy has thrown out dense clouds of high energy plasma. These observations may lead to a better understanding of the very energetic processes that appear to be occurring in galactic nuclei. It is reasonable to expect that the study of X-ray polarization will be no less rewarding than the corresponding optical and radio observations.

Methods of measuring X-ray polarization are limited by the interaction and subsequent loss of the incident photons in the analysing material. Coherent, or Bragg scattering from crystals provides an efficient means of observing X-ray polarization but is only effective at those wavelengths which satisfy the Bragg condition. When $2 \theta=90^{\circ}, \theta$ being the Bragg angle, only those photons with the proper wavelength and whose electric vector is perpendicular to the plane of incidence will be scattered. Photoelectric losses and the severe energy dependence limit the usefulness of this technique. Anomalous transmission, or the Borrmann effect, where photons with the proper energy and polarization are preferentially transmitted by a crystalline slab, can also be used. The low efficiency and narrow spectral response are again detrimental.

Incoherent scattering from free electrons provides an alternate way of detecting $\mathrm{X}$-ray polarization over a broad energy range. Only those photons with electric

\footnotetext{
* This work was supported in part by the National Aeronautics and Space Administration under Grants NGR-33-008-102, NGR-33-008-012, NGR-33-008-125 and Contract NAS 8-24668, and in part by the Air Force Office of Scientific Research under Grant AFOSR-70-1945. It is Columbia Astrophysics Laboratory Contribution No. 31.
} 
vectors normal to the plane of incidence are scattered at $90^{\circ}$. This anisotropy in the scattering cross section can be exploited to measure the polarization of the incident flux. The incoherent scattering cross section asymptotically approaches the Thomson cross section at low photon energies, and is essentially independent of wavelength until the photon energy becomes comparable to the electron rest mass energy. Incoherent scattering can be successfully exploited to measure polarization provided the binding energy of the atomic electrons in the scattering block is small compared to the incident photon energy. By choosing low $Z$ materials, photoelectric losses can be minimized.

X-ray polarimeters utilizing incoherent scattering have been constructed at this laboratory and used in astrophysical measurements on several occasions. Because of the relatively low intensity of extraterrestrial X-ray sources, as well as the low efficiency of the analysing technique, it is essential that the effective area of the instrument be maximized and that sources of background be reduced. The most efficient incoherent scattering material is hydrogen, but practicability necessitates that the scattering targets be made of lithium. In this material, the photoelectric and incoherent cross sections become comparable at about $5 \mathrm{keV}$, establishing the effective low energy cutoff of the instrument. Work is in progress to use lithium hydride, which should improve the low energy response. The shape of the scattering targets was established by demanding a high probability of a photon making at least one interaction in the block, but that the probability of a second scatter be small. The length of 5 in. was chosen, corresponding to one mean free scattering path. A cross section of 2 in. $\times 2$ in. was found to maximize the detected flux without causing a reduction in polarization sensitivity through multiple scattering.

Photons scattered out the sides of the blocks are detected by proportional counters. These detectors provide high efficiency over the energy range to be studied and also yield pulse amplitude proportional to the detected photon energy. The high energy limit for the polarimeter is determined chiefly by the rapid decrease in flux with increasing energy observed in most X-ray sources. For the Crab Nebula, for example, the incident flux at $25 \mathrm{keV}$ is only $4 \%$ of the flux at $5 \mathrm{keV}$. Counters with 25 mil Be entrance windows and 3 atmospheres of xenon gas filling were chosen to achieve maximum sensitivity in the 5 to $25 \mathrm{keV}$ range. The counters adjacent to each scattering block subtend a finite solid angle, allowing photons scattered at angles other than $90^{\circ}$ to reach the detectors. This effect dilutes the polarization sensitivity, but can be taken into account by calibration. Laboratory tests with polarized X-ray beams, and Monte Carlo calculations revealed that $100 \%$ polarized incident flux results in a detected counting rate asymmetry of $30 \%$. Monte Carlo calculations and laboratory measurements of the instrumental efficiency were also performed. The results are shown in Figure 1.

Cosmic ray background poses the biggest problem in detecting X-ray polarization. Several precautions have been taken to reduce the number of spurious counts. Since each detector contributes to the background rate, the polarimeter is constructed with two scattering blocks for each proportional counter, thereby doubling the signal-to- 
background ratio. The instrument consists of a matrix of scattering blocks and proportional counters, as shown in Figure 2. Further background reduction is achieved by surrounding the instrument with an anticoincidence shield sensitive to charged particles. Instruments already flown in sounding rocket experiments have used plastic scintillator anticoincidence shields. Gas-filled guard counters will also achieve the

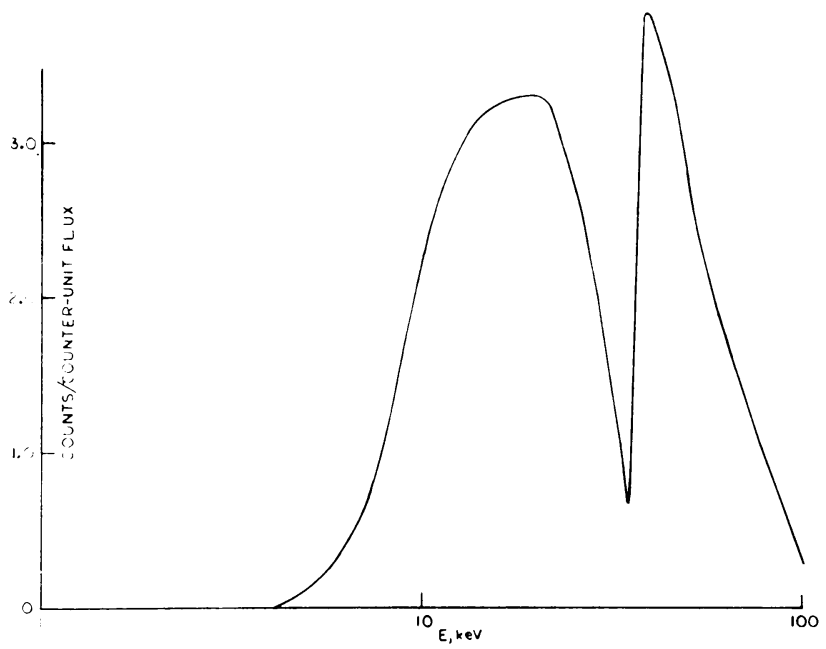

Fig. 1. Efficiency of the polarimeter versus energy.

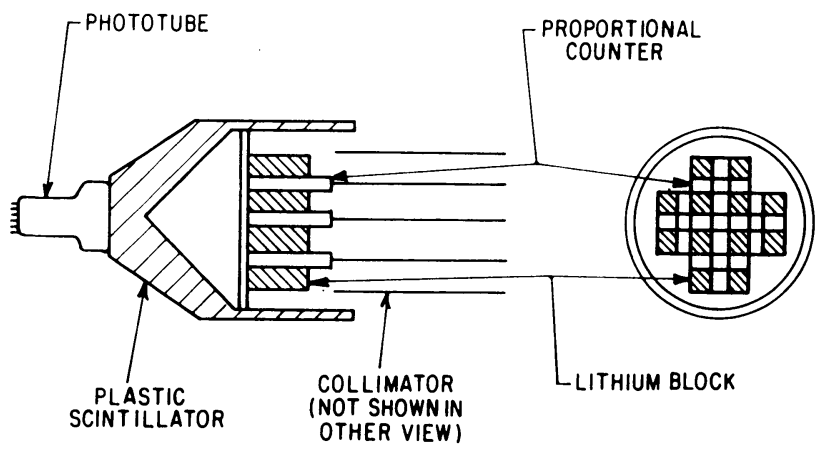

Fig. 2. Lithium polarimeter in the sounding rocket configuration.

same result, while adding less mass to the payload. The use of proportional counters allows pulse height analysis to be applied to eliminate those background events outside the 5 to $25 \mathrm{keV}$ energy range. Experience has shown that pulse height discrimination and anticoincidence vetoing reduce the background rate to $0.01 \mathrm{count} / \mathrm{cm}^{2} \mathrm{~s}$ keV counter.

The difference in pulse shape due to a photon and charged particle can be used to distinguish between $\mathrm{X}$-ray and cosmic ray events. Charged particles produce a long 
ionization track, and the risetime of the subsequent proportional counter pulse is slow, as charge from different points on the track must drift in towards the ancde. $\mathrm{X}$-ray events rise quickly, however, as all the photon energy is absorbed in a single photoelectric event. An electronic technique to recognize this difference and discriminate against the slowly rising pulses has been developed. The cosmic ray background was simulated using Compton electrons produced by the interaction of gamma rays from $\mathrm{Co}^{60}$ and $\mathrm{Cs}^{137}$ sources with the walls of the detectors. The laboratory tests revealed that between 30 and $60 \%$ of the gamma ray-induced events could be rejected at the expense of only 15 to $20 \%$ of the valid X-ray pulses. The results of one rocket flight showed that the overall background rate could be reduced to 0.0034 count $/ \mathrm{cm}^{2} \mathrm{~s}$ keV counter.

Versions of the incoherent scattering polarimeter have been incorporated in a balloon payload and three sounding rocket payloads. Further plans include another sounding experiment and satellite-borne instruments. The balloon flight was performed in November 1967, with a prototype instrument consisting of four scattering blocks and four detectors. The effectiveness of various background rejection methods was established. In addition, background effects which could introduce spurious polarization were investigated. No evidence of background-induced polarization was noted (Wing, 1968).

The first rocket-borne polarimeter experiment was performed in July 1968, in search of X-ray polarization from Sco X-1. The instrument was carried to a peak altitude of 99 miles by an Aerobee- 150 sounding rocket. Once above the atmosphere, the rocket was pointed at the X-ray star and the nose cone was ejected, exposing the lithium scattering blocks to the X-ray flux. The payload was then rotated around the line of sight at $1 \mathrm{rpm}$. X-ray polarization would then manifest itself as a modulation in the counting rate of each detector in phase with the payload rotation frequency. The data were analysed by fitting the counting rate to the equation

$$
R(t)=R_{0}\left(1+M_{1} \cos 2 \omega t+M_{2} \sin 2 \omega t\right)
$$

using the method of least squares. The background rate was established by pointing the instrument at an empty region of the sky during the second portion of the flight. After subtracting the background it was found that the signal modulation was finite. Since polarization is a positive definite quantity obtained as the quadratic sum of its components, random fluctuations due to a finite amount of data lead to a non zero result. In this experiment, the data obtained were statistically consistent with zero polarization. The outcome of the measurements of Sco X-1 can best be interpreted as establishing an upper limit on the polarization. Our analysis revealed that there is a $99 \%$ probability that the X-ray flux between 5 and $16.8 \mathrm{keV}$ is less than $18 \%$ polarized (Angel et al., 1969).

A second sounding rocket experiment was carried out to investigate X-ray polarization from the Crab Nebula (Tau X-1). Since the flux from this source is only 0.1 of that of Sco X-1, further steps were required to lower the background. Risetime discrimination circuits were added to the electronics and a factor of four reduction in 
background was anticipated from laboratory measurements. The flight occurred in March 1969, and all aspects of the experiment performed as planned. X-ray data were obtained for four minutes while viewing the source. The net background rate was not as low as anticipated, mainly due to the lack of correspondence between the laboratory simulation and the actual cosmic ray environment above the atmosphere. As before, the data were analysed in accordance with Equation (1) by the method of least squares. A result consistent with zero was obtained. With $99 \%$ confidence it can be stated that the X-ray flux from the Crab Nebula is less than $27 \%$ polarized between 5 and $22 \mathrm{keV}$ (Wolff et al., 1970).

A further use of the X-ray polarimeter is currently in progress to measure hard $\mathrm{X}$-ray polarization from solar flares. The energy range of the instrument has been extended to $80 \mathrm{keV}$ and the electronics modified to handle the high fluxes expected. The sensitivity of the instrument is sufficient to measure polarizations of less than $1 \%$ and also to examine the energy dependence of the anticipated polarization. Preliminary results of a satellite-borne solar flare polarimeter have already been reported (Tindo et al., 1970). Our experiment will be performed from a sounding rocket in October 1970. A second attempt to measure the polarization of the Crab Nebula is also under way. A larger, more sensitive polarimeter is under construction and will be flown in an Aerobee-350 (22-in. dia) sounding rocket in January 1971. The increase in effective area and viewing time will result in approximately three times as much data, establishing a lower limit of $10 \%$ on the polarization. A satellite version of the incoherent scattering polarimeter is being designed for use on the OSO I satellite.

A larger, more advanced polarimeter has been proposed for the HEAO satellite. A satellite-borne instrument has the essential advantage of long viewing periods precluded in sounding-rocket work. In this way it becomes feasible to examine the weaker but none-the-less interesting X-ray sources for polarization. The proposed instrument consists of a $12 \times 12$ array of $2^{\prime \prime} \times 2^{\prime \prime}$ square lithium and lithium hydride blocks, each 5 in. long. Proportional counters filled with three atmospheres of xenon gas are interspersed between the blocks. A gas guard counter surrounds the instrument to provide cosmic ray background suppression, as shown in Figure 3.

The large area and long viewing time afforded enable extremely sensitive measurements to be made. With an observing period of one day, a polarization as small as $0.3 \%$ could be detected in the total X-ray flux of the Crab Nebula with $99 \%$ confidence. The polarization sensitivity to the Crab Nebula X-ray pulsar is $2 \%$ under the same circumstances. It is important to note that the observed optical polarization of the nebula is $19 \%$ and the pulsar optical polarization at peak intensity is $10 \%$. A measurement of the X-ray polarization of M 87 could also be accomplished, the $3 \sigma$ limit in a one-day viewing period being $5 \%$. In only one orbit the polarization of Sco X-1 could be determined to less than $1 \%$. Such an instrument could easily be used to study recently reported X-ray flare stars as well.

The broad energy range of the polarimeter can be used to study the wavelength dependence of X-ray polarization. Photons produced by the synchrotron mechanism will be plane-polarized perpendicular to the magnetic field in the emission region, 


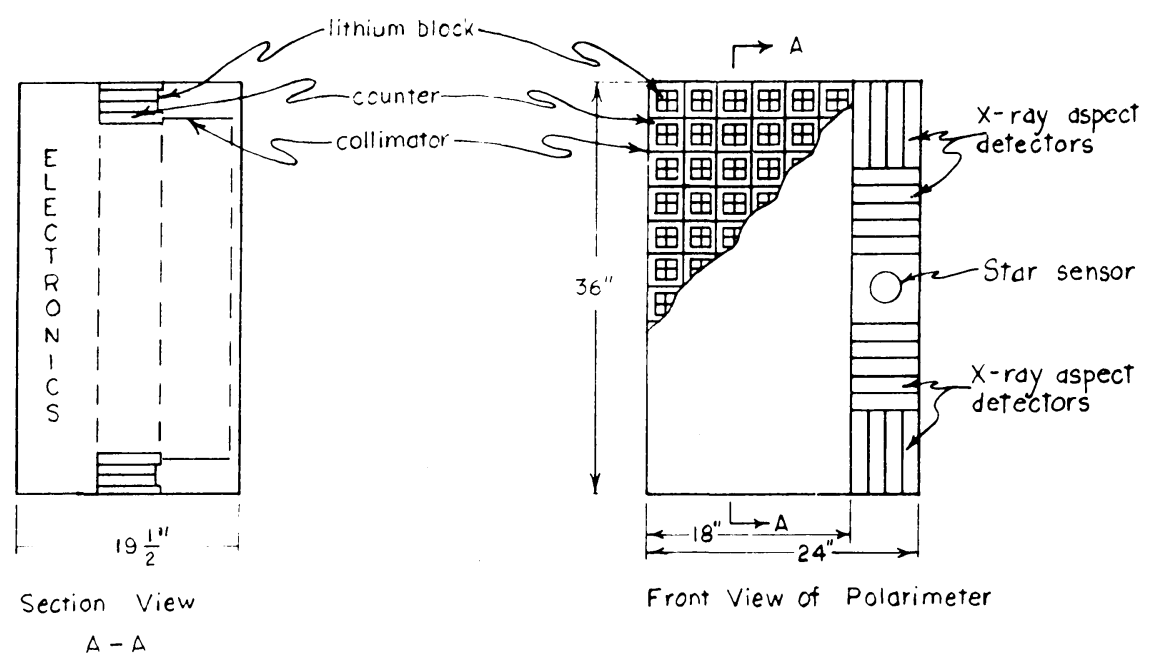

Fig. 3. Proposed design of lithium polarimeter for HEAO satellite.

independent of energy. $\mathrm{X}$ rays produced by linear bremsstrahlung, however, will exhibit polarization dependent on the photon energy. At short wavelengths, the polarization will be parallel to the plane of viewing, but at low energy the polarization is perpendicular. Such a mechanism has been proposed to explain solar flare X-ray bursts. Energy sensitive polarization measurements possible with the proposed instrument would serve to distinguish between these two emission processes.

\section{References}

Angel, J. R. P., Novick, R., Vanden Bout, P., and Wolff, R. S.: 1969, Phys. Rev. Letters 22, 861.

Tindo, I. P., Ivanov, V. D., Mandel'stam, S. L., and Shuryghin, A. I.: 1970, Solar Phys. 14,

Wing, T.: 1968, Ph.D. Dissertation, Columbia University, New York, N.Y.

Wolff, R. S., Angel, J. R. P., Novick, R., and Vanden Bout, P.: 1970, Astrophys. J. Letters 160, L21. 